\title{
Erfahrungen mit der Zertifizierung Medizinischer Laboratorien
}

\section{Gudrun Stamminger}

Zentrum für Diagnostik GmbH am Klinikum Chemnitz, Deutschland
Medizinische Laboratorien erfüllen eine Kernaufgabe innerhalb der Krankenversorgung und sind tragendes Element der Diagnostik, auch im Bereich der Notfallversorgung. Sie erweisen sich zunehmend als Schlüsselfaktor für kurze stationäre Liegezeiten und sind an der Konzeption und Realisierung einer rationalen Diagnostik unmittelbar beteiligt. Insbesondere im Bereich der Molekularen Diagnostik sind Laboratorien innovativ tätig und erfahren dadurch eine Aufwertung ihrer Tätigkeit weit über ihr bisheriges Feld hinaus. Labormediziner übernehmen die Interpretation komplexer Befunde, die therapeutische Beratung in der Infektiologie, Hämostaseologie und Transfusionsmedizin und haben beratende Funktionen im Hygienemanagement und bei der Erstellung von Resistenzstatistiken. Nicht zuletzt ist die Führung des Blutdepots Aufgabe des Labors. Zentrale Elemente unseres Berufsverständnisses sind Qualitätssicherung und umfassendes Qualitätsmanagement, zwei Bereiche, in denen wir uns durchaus als Vorreiter sehen.

Bei der Akkreditierung medizinischer Laboratorien werden nicht nur die laborinternen Abläufe begutachtet, sondern der komplette, die Qualität determinierende Prozess von der Probeneinsendung bis hin zur Befundinterpretation (Abb. 1).

Das Zentrum für Diagnostik GmbH wurde 2006 als 100\%iges Tochterunternehmen des Klinikums Chemnitz gegründet und erbringt außer für das Klinikum Chemnitz die kompletten Laborleistungen für 6 weitere sächsische Kliniken. Die Bettenkapazität dieser Häuser beträgt insgesamt etwa 3500, dabei umfasst das Labor-Leistungsangebot etwa 700 verschiedene Untersuchungen. Wir führen mit derzeit 165 Mitarbeitern (davon 8 Ärzte) rund 6 Millionen Untersuchungen pro Jahr durch.

Das Diagnostik-Zentrum ist zusammen mit der Klinikum Chemnitz GmbH durch die Joint Commission International Chicago akkreditiert. Ferner haben wir uns 2009 einer eigenen Akkreditierung durch die Deutsche Gesellschaft für Akkreditierung GmbH (DGA) - jetzt Deutsche Akkreditierungsstelle (DAkkS) - unterzogen. Unsere Laborstandorte

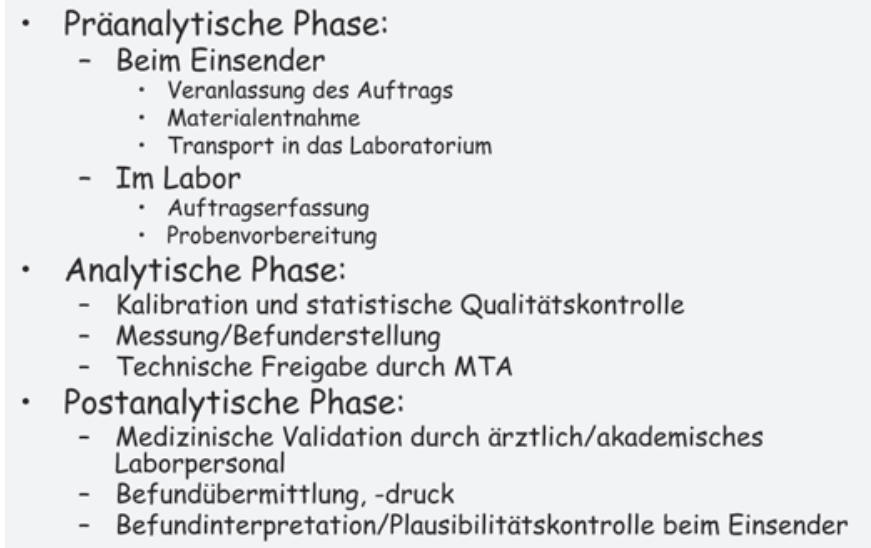

Abb. 1. Determinanten der Qualität in der Labormedizin.

in Chemnitz, Aue, Oschatz und Schneeberg sind damit nach DIN EN ISO 15189 akkreditiert und besitzen die Kompetenz auf den Untersuchungsgebieten Mikrobiologie, Virologie, Klinische Chemie, Immunologie, Humangenetik und Transfusionsmedizin. Die Akkreditierung bezieht sich somit auf alle Untersuchungsgebiete, in denen das Zentrum für Diagnostik GmbH derzeit Laborleistungen erbringt.

\section{Was bringt die Akkreditierung?}

Medizinische Laboratorien müssen nach den Richtlinien der Bundesärztekammer zur Qualitätssicherung in medizinischen Laboratorien ein umfassendes Qualitätsmanagement betreiben und dokumentieren; insofern ist die Frage nach dem Nutzen einer zusätzlichen - freiwilligen - Akkreditierung durch die DAkkS durchaus berechtigt. Wir sehen darin allerdings eine Reihe von Vorteilen (Abb. 2), die sich wie folgt darstellen:

\begin{tabular}{ll}
\hline KARGER & (c) 2012 S. Karger GmbH, Freiburg \\
0378-584X/12/3516-0015\$38.00/0 \\
Fax +49 7614520714 \\
$\begin{array}{l}\text { Information@Karger.de } \\
\text { www.karger.com }\end{array}$ & $\begin{array}{l}\text { Accessible online at: } \\
\text { www.karger.com/onk }\end{array}$
\end{tabular}


Eine schnelle, sichere und schonende Diagnostik, die der Gesundheit und der Gesundheitserhaltung der Patienten dient, verstehen wir als Grundlage der modernen Medizin. Ferner haben wir uns konkrete, messbare Qualitätsziele gesetzt, die unseren Anspruch an eine leistungsstarke, kundenund wettbewerbsorientierte Labordiagnostik unterstreichen (Abb. 3).

Die Zentrum für Diagnostik GmbH nimmt an der von den nationalen Ringversuchsorganisationen (INSTAND e.V. und Deutsche Vereinte Gesellschaft für Klinische Chemie und Laboratoriumsmedizin e.V. (DGKL)) organisierten externen Qualitätssicherung teil und ist ein von der Bundesärztekammer berufenes Sollwertlaboratorium für die externe Qualitätskontrolle bei INSTAND.

- Objektiver Nachweis der Güte der erbrachten Leistungen

- Kompetenz nach internationalen Standards wird ausgewiesen.

- Das Vertrauen der Einsender in die Qualität der erbrachten Leistungen wird gestärkt.

- Wichtiges Kriterium für die Auswahl als Dienstleister (z.B. Fremdleistungen für andere Laboratorien) - daher Wettbewerbsvorteil

- Voraussetzung für die Auswahl als Prüflabor bei vielen Studien und im Rahmen von Zentren

- Zukünftig Voraussetzung für die Anerkennung als Sollwertlaboratorium

Abb. 2. Vorteile einer Akkreditierung.

Qualität der Ergebnisse:
$\checkmark \quad$ Erfüllung der Kriterien der BÄK
$\checkmark \quad$ Minimale Fehlerquote bei Ringversuchen (2011: 98,6\%
Bestehensquote)
$\checkmark \quad$ Sollwertlaboratorium bei INSTAND
Hohe Einsenderzufriedenheit:
$\checkmark \quad$ Einhaltung festgelegter Bearbeitungszeiten
$\checkmark \quad$ Stabilisierung des Laborinformationssystems
$\checkmark \quad$ Organisation von 5 Einsenderschulungen pro Jahr
Hohe Mitarbeiterzufriedenheit:
$\checkmark \quad$ Minimierung von Mehrarbeit gegenüber dem Vorjahr
$\checkmark \quad$ (Personalpolitik)
$\checkmark$ Teilnahme an mind. 2 Fortbildungen/a

Abb. 3. Qualitätsziele im Rahmen der Akkreditierung.
Diese Qualitätsansprüche bestehen unabhängig von jedweden Akkreditierungsvorhaben. Darüber hinausgehende Ziele sind betriebswirtschaftlicher Art, etwa das Erreichen eines positiven Betriebsergebnisses und die Sicherung der Liquidität durch Rechnungslegung innerhalb von 4 Wochen nach Leistungserbringung. Gute Kommunikation und regelmäßiger Erfahrungsaustausch festigen die vertrauensvolle Zusammenarbeit mit Geschäftspartnern und Kollegen und sind eine notwendige und qualitätssichernde Maßnahme. Nicht zuletzt ist die Einhaltung von Bestimmungen des Arbeits-, Gesundheits- und Brandschutzes ein Teil des Qualitätskonzeptes.

Die Verantwortlichkeit für die Qualität der labormedizinischen Untersuchungen liegt bei der Geschäftsleitung, die sich in unserem Zentrum aus Geschäftsführerin, Ärztlicher Leiterin, Abteilungsleitungen sowie leitender Medizinisch-Technischer Assistentin (MTA) zusammensetzt. Wir haben außerdem eine Stabsstelle mit einer hauptamtlichen Beauftragten für das Qualitätsmanagement eingerichtet, die sämtliche Qualitätsmanagementprozesse überwacht. Als sehr hilfreich hat sich die Implementierung eines elektronischen Qualitätsmanagement-Systems (QM-System) erwiesen. Es handelt sich um ein datenbankgestütztes System, das die Verwaltung und Archivierung von Dokumenten übernimmt, alle Änderungen automatisch registriert und über ein hinterlegtes Rechte- und Rollenkonzept verfügt. Die Freigabe von Dokumenten erfolgt über einen direkt integrierten Freigabe-Workflow und bietet vielfältige Möglichkeiten zur Dokumentenrecherche und -kontrolle. Das System lässt sich gut für Maßnahmen zur Erfolgskontrolle nutzen, etwa zur Erfassung der Zufriedenheit von Einsendern. Das in Führungs-, Haupt- und Serviceprozesse untergliederte QM-System erlaubt raschen Zugriff auf sämtliche relevanten Materialien wie Arbeitsanweisungen und Einsenderinformationen. Dadurch lassen sich mit relativ wenig Aufwand Maßnahmen zur kontinuierlichen Qualitätsverbesserung konzipieren und dokumentieren. So haben wir in unserem Zentrum ein Projekt zur Optimierung der laborinternen Turn-around-Time (TAT) für Vitalparameter aufgelegt und im Wesentlichen durch Schulungsmaßnahmen und Verbesserung der Gerätetechnik erfolgreich umsetzen können (Abb. 4).
Abb. 4. Beispielprojekt zur Qualitätsverbesserung: Optimierung der Turn-around-Time für Vitalparameter.
- Infolge der Zentralisierung und Konsolidierung der Analytik an einem Standort im Klinikum kam es zur Verlangsamung der Ergebniszeiten - relevant für Vitalparameter

- Konsequenz: Forderung nach mehr (teurer) POCT-Analytik seitens der Kliniken

- Unsere Antwort: 2009 - Projekt zur Verbesserung der laborinternen TAT-Zeiten

- Beispielhafte Maßnahmen:

- Analyse der tatsächlichen Zeiten über das Labor-Informationssystem (Ausgangspunkt und seither monatliche Auswertung)

- Ausschöpfung des organisatorischen Optimierungspotentials ohne zusätzliche Arbeitskräfte

- Schulung des Stationspersonals zur Verbesserung in der Präanalytik (Abnahme Kennzeichnung von Proben, Anforderungen usw.)

- Optimierung in der Gerätetechnik 
- Anmeldung bei der zuständigen Akkreditierungsstelle (DAkkS $\mathrm{GmbH}$ ) - welche Bereiche sollen akkreditiert werden?

- Einreichen aller relevanten Dokumente (z.B. Fragebögen, QMHandbuch, Dokumente zu Prüfverfahren usw....)

- Vereinbarung über einen Auditzeitraum durch Begutachter (System- und Fachgutachter) in Abhängigkeit von der Anzahl der Fachbereiche

- Durchführung des Audits

- Auswertung und Besprechung der festgestellten Abweichungen von der Norm

- Einreichen der Korrekturmaßnahmen durch Antragsteller

- Übergabe der Akkreditierungsurkunde

- Festlegung des Überwachungsplanes

Abb. 5. Ablauf einer Erstakkreditierung nach DIN EN ISO 15189 im Laboratorium.

An den Fremdversand nicht selbst erbrachter Leistungen stellen wir ebenfalls hohe Qualitätsanforderungen. Im QMSystem wurde dafür ein Punktesystem hinterlegt, das als Entscheidungskriterium für die Laborwahl herangezogen wird. Dabei fließt der Nachweis einer Akkreditierung mit einem Anteil von $30 \%$ in die Gesamtbewertung ein; positiv können sich auch die Transportlogistik, die Art der Befundübermittlung und die Preisgestaltung auswirken.

Ein Beispiel für Optimierungsmöglichkeiten im Serviceprozess bietet das Fehlermanagement. So haben wir die Fehlerquelle «Hämolyse von Laborproben» durch Auswertung und Vergleich der Hämolyse-Häufigkeiten in den verschiedenen Fachabteilungen dokumentiert und dadurch Lösungsansätze zur Fehlerminimierung abgeleitet. Ein organisationsbezogener Fehler ist der Verfall von Blutprodukten im Labor, was durch Verbesserung der Logistik- und Abgabepraxis jedoch weitgehend vermieden werden kann. So ist es uns unter anderem durch Rücknahme nicht transfundierter Produkte ins Blutdepot unter Einhaltung der erforderlichen Lagerungsbedingungen sowie durch permanente Überwachung der Daten mithilfe eines IT-Systems gelungen, die Verfallquote seit vielen Jahren bei unter 1\% zu halten.

\section{Ablauf einer Erstakkreditierung}

Unser Entschluss, alle Bereiche des Zentrums für Diagnostik GmbH am Klinikum Chemnitz nach DIN EN ISO 15189 akkreditieren zu lassen, fiel im Jahr 2008. Nach entsprechenden Vorüberlegungen und Vorarbeiten (Abb. 5) erfolgten im Jahr 2009 (sowie für weitere Standorte im Jahr 2010) die Akkreditierung sowie die Festlegung eines Überwachungsplanes. Die Re-Akkreditierung sämtlicher Bereiche steht für das Jahr 2014 an.

Die im Rahmen der fortlaufenden Überwachungen dokumentierten Fehler und Abweichungen können system- oder fachbezogen sein. Wir konnten die in unserem Zentrum festgestellten Mängel jeweils kurzfristig beheben und dadurch das System weiter optimieren. Nach unserer Erfahrung muss Qualitätsmanagement von den Mitarbeitern als selbstverständlich empfunden werden und nicht als lästiges Beiwerk. Das QM-System ist Teil der täglichen Arbeit; so fordern wir zum Beispiel alle Mitarbeiter elektronisch zur Kenntnisnahme relevanter Dokumente auf. Eine zur «Qualitätsbeauftragen (TÜV)» fortgebildete Mitarbeiterin achtet darauf, dass Abweichungen von den Regeln möglichst sofort beseitigt werden. Wir legen Wert auf Regelmäßigkeit bei der Auswertung unserer Qualitätsziele sowie bei der Durchführung interner und externer Audits. Letztlich sehen wir den höheren Anspruch, den die öffentliche Wahrnehmung als akkreditiertes Labor mit sich bringt, als Ansporn zu permanenter Verbesserung. So werden wir im Jahr 2012 ein elektronisches Materialwirtschaftssystem einführen und den Informationsaustausch weiter vorantreiben. Schließlich hoffen wir, durch die geplante räumliche Zusammenführung aller Abteilungen unter einem Dach die Arbeit noch effektiver gestalten zu können.

\section{Disclosure Statement}

Es bestehen keine Interessenkonflikte. 